\title{
Comparison of spatio-temporal gait parameters according to shoe types in chronic stroke survivors: a preliminary study
}

\author{
Soung Kyun Hong ${ }^{a}$, Su Ho Park ${ }^{a}$, Sung Ri Shin ${ }^{b}$, Dong Geon Lee ${ }^{a}$, Seung Hoo Lee ${ }^{a}$, Sun Hye Jung ${ }^{a}$, \\ Seung Hyeon Pyo ${ }^{a}$, Kyeong-Bong Lee ${ }^{c}$, Gyu Chang Lee ${ }^{b}$ \\ ${ }^{a}$ Department of Physical Therapy, The Graduate School of Kyungnam University, Changwon, Republic of Korea \\ ${ }^{b}$ Department of Physical Therapy, College of Health Sciences of Kyungnam University, Changwon, Republic of Korea \\ ${ }^{\mathrm{c}}$ Physical Therapy Part, Department of Physical and Rehabilitation Medicine, Samsung Medical Center, Seoul, Republic of Korea
}

Objective: The purpose of this study was to investigate the impact of wearing various types of shoes on gait ability in stroke survivors and in order to gain information in regards to shoes that could possibly replace ankle orthosis.

Design: Cross-sectional study.

Methods: Eight hemiplegic survivors diagnosed with stroke participated in the study. Gait was analyzed using the GAITRite Electronic Walkway (CIR System Inc., USA) when subjects walked with no showed, walked with non-ankle-covered shoes, and walked with ankle-covered shoes. This study collected gait variables, including velocity, cadence, step length, stride length, single support time, and double support time, respectively.

Results: In the comparison of walking with no shoes, non-ankle-covered shoes, and ankle-covered shoes, there were significant differences in gait velocity, step length, stride length, and the less affected side single support time $(p<0.05)$. However, there were no significant differences in cadence, affected side single support time, and double support time.

Conclusions: Ankle-covered shoes had a positive impact on the gait of stroke survivors. However, it is necessary to conduct more studies comparing various types of shoes with ankle orthoses.

Key Words: Gait, Shoes, Stroke

\section{Introduction}

Stroke is the third leading cause of adult death and one of the major disabilities in the world [1]. According to the National Statistical Office, the number of stroke survivors is 102,529 in total, with 56,339 men and 46,190 women [2]. Hemiplegia due to stroke leads to asymmetric weight load [3], and particularly in standing position, the survivors suffer from an imbalance due to supporting $60 \%$ to $90 \%$ of their weight by leaning onto the less affected side. In addition, in the condition of standing still, the distribution of foot pressure shows significant forward and outward tendencies. To compensate for this, the ankle strategy is used to maintain balance so that the ground reaction force mainly acts on the foot that is not paralyzed [4]. Consequently, this imbalance has a negative impact on gait ability, and addressing this problem is a very important goal in rehabilitation [5]. To solve problems related to the stroke survivors' balance and gait, various methods of intervention, including ankle orthoses, functional electrical stimulation or a control type gait auxiliary devices are used.

An orthosis can control foot drop in the swing phase by providing increased resistance against ankle plantar flexion, and it was reported that limiting ankle plantar flexion can

Received: 12 February, 2018 Revised: 20 March, 2018 Accepted: 20 March, 2018

Corresponding author: Gyu Chang Lee (ORCID http://orcid.org/0000-0001-6404-203X)

Department of Physical Therapy, Kyungnam University, 7 Kyungnamdaehak-ro, Masanhappo-gu, Changwon 51767, Republic of Korea Tel: 82-55-249-2739 Fax: 82-505-999-2173 E-mail: leegc76@hanmail.net

(c) This is an Open-Access article distributed under the terms of the Creative Commons Attribution Non-Commercial License (http://creativecommons.org/licenses/ by-nc/4.0) which permits unrestricted non-commercial use, distribution, and reproduction in any medium, provided the original work is properly cited.

Copyright $@ 2018$ Korean Academy of Physical Therapy Rehabilitation Science 
have a positive impact on gait velocity and effectiveness [6]. In addition, it was also reported that using an orthosis improved balance in gait [7]. However, since orthoses limit the movement of the joint, it may prevent normal function of the ankles [8]. Since it is relatively large and is applied by directly coming in contact with the lower limbs, it is inconvenient to wear and not cosmetically pleasing. Also, the production process is complex rendering it to be expensive. In addition, since the survivors are required to wear shoes with use of ankle orthoses, it is cumbersome to purchase shoes that are larger than the actual foot size of the stroke survivor. [8].

It is also necessary to conduct studies of the shoes that could supplement and replace these problems related to ankle orthoses. Currently, most studies have been conducted on healthy adults, and studies focused on shoes that may serve to replace ankle orthoses in stroke survivors with hemiplegia are insufficient. Several other studies have reported that raising the shoe height on the healthy side with shoe lifts and wedges increased symmetrical weight bearing on the affected side $[9,10]$, but it has the inconvenience of having to wear a larger size shoe simliar to an ankle orthosis. The Masai Barefoot Technology (MBT) shoe is a functional shoe that provides stability to the ankle joint and has a curved surface at the bottom of the shoe, which is excellent for upright walking and shock absorption [11]. The minimalist shoes are lightweight shoes with thin soles, which increase the activation of muscular function on the plantar side [12], and there is an increase in walking ability [13]. Since the MBT shoes have a curved surface structure and the minimalist shoes cannot wrap aroudn the ankle joints both shoe types have difficulty in increasing ankle stability for stroke survivors with hemiplegia. The ankle-covered shoes are similar to ordinary shoes, but they have a structure that covers the upper part of the ankle joint and the stability of the ankle, which has a similar advantage as the MBT shoes but the floor is not curved. Subsequently, it can provide more stability to patients, and has other advantages in which they are easy to wear and are inexpensive. Therefore, patients with chronic hemiplegic stroke who present with slight instability of the ankle joint may be offered these shoes as an alternative to wearing orthoses.

The purpose of this study was to investigate the effects of walking with no shoes, non-ankle-covered shoes, and ankle-covered shoes in order to replace the ankle orthoses in persons with hemiplegic chronic stroke.

\section{Methods}

\section{Participants}

This study was conducted with hemiplegic stroke survivors. The subjects were recruited through the bulletin board at Gupo Bumin Hospital in Busan and were selected according to the following selection criteria: 1) persons who had received a diagnosis of stroke at least 6 months ago; 2 ) those who had a score higher than 21 on the Mini-Mental Status Examination-Korea; 3) those who could independently walk more than 10 meters, regardless of the use of an assistive device; 4) those who did not have any orthopedic diseases on the lower limbs; and 5) those who did not have any problems in visual and auditory functions. Finally, eight hemiplegic stroke survivors were selected, and the basic characteristics of the subjects are as follows: There were six men and two women with an average age of $65.38 \pm 8.32$ years, average height of $168.38 \pm 5.88 \mathrm{~cm}$, average weight of $68.63 \pm 10.2 \mathrm{~kg}$, and average shoe size of $253.75 \pm 10.94 \mathrm{~cm}$. The affected side was the right in six persons and the left in two persons. The participants were thoroughly informed about the purpose and procedures of the study before voluntarily signing the consent form. This study was conducted after receiving approval from the Kyungnam University Ethics Committee (Approval No. 1040460-A-2017-036).

\section{Procedures}

The main experiment was conducted after collecting data on the research subjects' sex, age, height, weight, paralyzed part, and shoe size. The subjects underwent the three various gait conditions, including the no shoes condition, the nonankle-covered shoes condition, and the ankle-covered shoes condition (Figure 1). The conditions were randomly chosen by the research assistant. For gait analysis of each condition, the GAITRite Electronic Walkway (CIR System Inc., Natick, MA, USA) was used. Of the gait-related data collected through GAITRite Electronic Walkway, the spatio-temporal variables (gait velocity, cadence, step length, single support time, and double support time, etc.) were analyzed and then compared [14]. In each condition, gait was repeatedly measured three times. Between each measurement, a 3-minute break was provided, and between each condition, a 5-minute break was provided, considering the time for putting on shoes. For subject safety, two research assistants were in close proximity to and carefully observed the subjects during the progress of the experiment. 


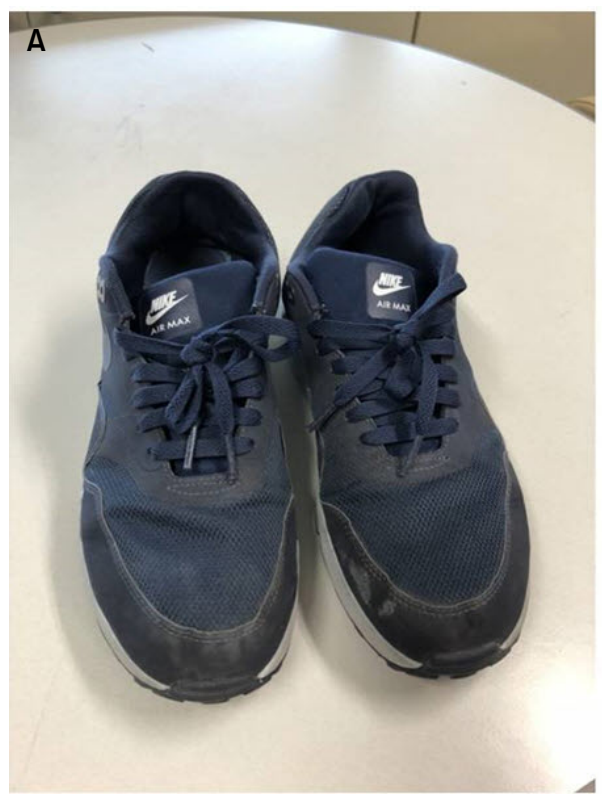

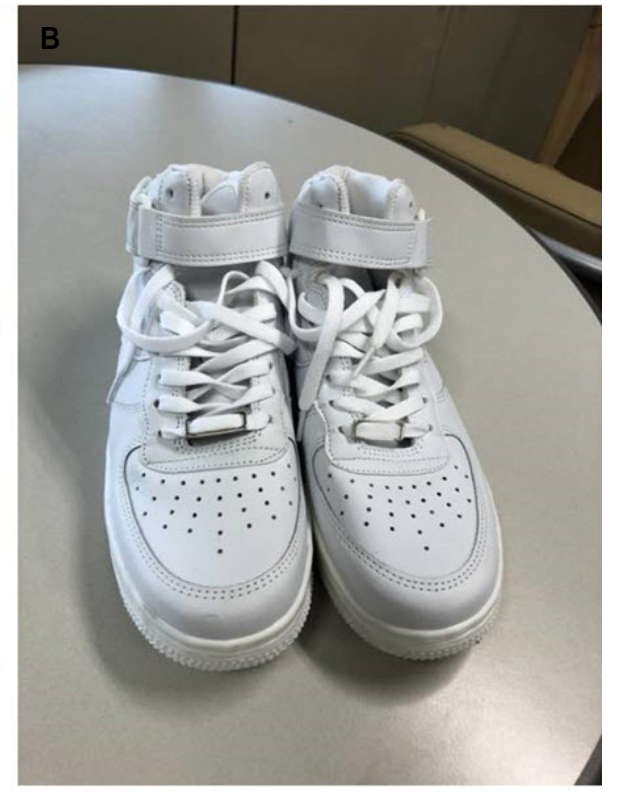

Figure 1. The different types of shoes. (A) Non-ankle-covered shoes. (B) Ankle-covered shoes.

Table 1. Comparison of spatiotemporal gait parameters among three conditions

\begin{tabular}{lcccc}
\hline \multicolumn{1}{c}{ Variable } & No shoes & Non-ankle-covered shoes & Ankle-covered shoes & $p$-value \\
\hline Velocity (m/s) & $59.69(21.64)$ & $66.70(19.58)$ & $68.95(21.97)$ & 0.01 \\
Cadence (step/min) & $89.75(13.56)$ & $96.11(12.19)$ & $93.91(13.11)$ & 0.09 \\
$\quad$ Step length (cm) & & & & \\
$\quad$ Affected & $38.74(11.2)$ & $41.13(10.29)$ & $43.28(11.78)$ & 0.01 \\
$\quad$ Less affected & $40.16(10.5)$ & $42.25(11.34)$ & $44.39(11.45)$ & 0.00 \\
Stride length (cm) & & & & 0.00 \\
$\quad$ Affected & $79.22(21.29)$ & $83.40(21.24)$ & $87.54(22.01)$ & 0.00 \\
$\quad$ Less affected & $79.34(21.46)$ & $83.65(20.9)$ & $88.53(22.39)$ & 0.14 \\
Single support time (s) & $0.39(0.06)$ & $0.36(0.03)$ & & $0.39(0.04)$ \\
$\quad$ Affected & $0.43(0.05)$ & $0.40(0.05)$ & $0.43(0.05)$ & 0.02 \\
$\quad$ Less affected & $0.53(0.24)$ & $0.49(0.14)$ & $0.46(0.15)$ & 0.20 \\
Double support time (s) & $0.52(0.23)$ & $0.49(0.13)$ & $0.47(0.15)$ & 0.42 \\
$\quad$ Affected & & & \\
$\quad$ Less affected & & & & \\
\hline
\end{tabular}

Values are presented as mean (SD).

\section{GAITRite}

The GAITRite Electronic Walkway is a gait analysis system that has an effective measurement area with a width of $61 \mathrm{~cm}$ and a length of $366 \mathrm{~cm}$, in which sensors are arranged in the form of a grid $(48 \mathrm{~cm} \times 288 \mathrm{~cm})$, located at an interval of $1.27 \mathrm{~cm}$. Subjects walk on a gait mat that is spread out on a flat floor. A sensor responds to the foot pressure, and the information is saved on a computer through a serial interface cable to collect the information about the spatio-temporal gait variables [15]. All subjects performed each condition at a comfortable gait velocity for more than 6 meters, including the 4 meters-long gait mat.

\section{Data analysis}

For a statistical analysis of all data, SPSS ver. 12.0 (SPSS Inc., Chicago, IL, USA) was used. To examine the demographic characteristics of the subjects of this study, a descriptive statistical analysis was conducted. The collected data on participants' GAITRite spatiotemporal variables were compared and analyzed according to each condition using the Friedman analysis of variance test. In addition, the Wilcoxon signed-rank test was used for post-hoc analysis. Statistical significance level was specified at $\alpha=0.05$. 


\section{Results}

The results of this study are shown in Table 1. In the comparison of the subjects' walking with no shoes, non-anklecovered shoes, ankle-covered shoes, there were significant differences in gait velocity $(p<0.05)$, the affected side step length $(p<0.05)$, the less affected side step length $(p<0.05)$, the affected side stride length $(p<0.05)$, the less affected side stride length $(p<0.05)$, and in the less affected side single support time $(p<0.05)$. However, there were no significant differences in cadence, affected side single support time, affected side double support time and the less affected side double support time. In the post-hoc analysis there were significant differences in the two types of shoes compared with no shoes $(p<0.05)$, except for the less affected single support time.

\section{Discussion}

This study compared differences in spatio-temporal gait variables according to three conditions, including the condition of no shoes, the non-ankle-covered shoes, and the ankle-covered shoes in hemiplegic stroke survivors. As a result, in the comparison of no shoes and ankle-covered shoes, the velocity of ankle-covered shoes condition increased by $9.26 \mathrm{~m} / \mathrm{s}$. In the comparison of the no shoes and non-ankle-covered shoes condition, the velocity of the non-ankle-covered shoes increased by $7.01 \mathrm{~m} / \mathrm{s}$. In the comparison of the no shoes and ankle-covered shoes condition, the affected side step length and less affected side step length of the ankle-covered shoes condition increased by 4.54 and $4.23 \mathrm{~cm}$. In the comparison of no shoes and non-ankle-covered shoes, the affected side step length and less affected side step length of non-ankle-covered shoes increased by 2.39 and $2.09 \mathrm{~cm}$ respectively, and there was a statistically significant difference. Additionally, in the comparison of the no shoes and ankle-covered shoes condition, the affected side stride length and the less affected side stride length of ankle-covered shoes condition increased by 8.32 and 9.19 $\mathrm{cm}$ In the comparison of no shoes and non-ankle-covered shoes condition, the affected side stride length and less affected side stride length of the non-ankle-covered shoes increased by 4.18 and $4.31 \mathrm{~cm}$ respectively, and there was significant difference. In the less affected side single support time, there was no statistically significant difference between ankle-covered shoes and no shoes walking conditions. Howver, walking with non-ankle covered shoes de- creased by 0.03 seconds compared to the no shoes walking condition, and there was statistically significant difference. In the comparison of the subjects' walking with no shoes, non-ankle-covered shoes, and ankle-covered shoes condition, there were no significant differences in cadence, affected side single support time, and double support time. There was a significant difference of the two types of shoes compared with the no shoes condition except for the less affected single support time in post-hoc analysis. Comparing the ankle-covered shoes with the non-ankle-covered shoes condition, there were no significant differences in all conditions except for the less affected side single support time. However, in the ankle-covered shoes condition, compared to walking with no shoes and non-ankle-covered shoes, gait ability was better than the no shoes and the non-ankle-covered shoes condition except for the single support time. It can be judged through these results that the ankle-covered shoes provided a relative amount of stability and had a positive impact on gait ability.

Gait is a complex process in which the nervous and musculoskeletal systems of the human body are used in complicated ways [14]. Stroke survivors show abnormal gait patterns due to asymmetrical postures due to hemiplegia [15], and in particular, the stance phase of the affected side becomes shorter in the gait cycle while the swing phase becomes longer [16]. Similarly, in this study, when the subjects did not put on shoes, the affected side single support time was 0.39 seconds, which was shorter than the less affected side single support time, and the stance phase on the affected side was short while swing phase was long. However, with the application of non-ankle-covered and ankle-covered shoes, there was a statistically significant difference between the stance phase and swing phase, and also in the ankle-covered shoes condition, the subjects had larger step length and stride length values, had the same stance phase and swing phase duration compared to walking with no shoes, which meant that the ankle-covered shoes provided greater stability compared to wearing no shoes. In addition, when subjects walked with the non-ankle-covered shoes, they exhibited a shorter stance phase, step length and stride length on the affected side when they walked in the ankle-covered shoes, which showed that the ankle-covered shoes were more stable than non-ankle-covered shoes and was more effective for gait.

Putting on an ankle orthosis has a positive impact on the gait variables of hemiplegic stroke survivors and brings about significant changes in the variables, including gait ve- 
locity, gait asymmetric index, and functional balance control [17], as well as improvements in overall gait velocity, effectiveness and gait pattern [18]. In addition, it was reported that the use of an ankle orthoses in stroke survivors increased the stability of the ankle joint in the stance phase, which would lead to the increase in gait velocity [19]. In addition, Rao et al. [17] noted that putting on an ankle orthosis greatly improved gait velocity, step length, stride length and double support time.

The results of this study were consistent with those of the previous studies in which the ankle-covered shoes showed the highest results in both gait velocity, step length, stride length and double support time than walking with no shoes and non-ankle-covered shoes. It is judged that the anklecovered shoes provided ankle stability by in a similar manner to an ankle orthosis. However, since ankle orthoses may cause skin problems, produce discomfort with having to put on shoes again after putting on an orthosis, and limit the movement of the joint, orthoses have limitations in producing less than normal ankle function and the burden cost [20]. Ankle-covered shoes can reduce ankle joint instability similar to the effects of an ankle orthosis, have an effect on gait improvement, and are reasonably priced, and are more comfortable to wear. Not all patients are able to wear ankle braces after a stroke. Depending on the degree of improvement of various symptoms such as ankle stiffness, strength, and paralysis, ankle assistance should be applied appropriately. If patients present with severe after effects post stroke, they may need to purchase an orthosis immediately. In case of weakness, stiffness, and paralysis, it is difficult to purchase an ankle orthosis every time depending on improvement of symptoms. Therefore, the experiment in this study evaluated the gait of persons with hemiplegic stroke with the shoes only and no orthoses. Although there were significant difference in the shoe conditions compared to the no shoes conditions, the non-ankle-covered shoes condition showed no significant difference in all variables except for the less affected side single support time. Therefore, it cannot clearly demonstrate the difference between the types of shoes. Eckhardt et al. [21] reported that a comparison of normal shoes with the Orthopedic shoe that covers the ankle showed a $22 \%$ reduction in the Timed Up and Go test (dynamic balance), an increase in gait velociy of $37 \%$, and an increase in cadence of $17 \%$. In this study, the walking speed of subjects wearing the ankle-covered shoes was the greatest. These results could provide a basic guideline for shoes worn in everyday life after the initial stroke incident. However, these shoes also come with some limitations; they cannot improve foot drop, inversion or eversion. However, they can reduce ankle joint instability and sway. Therefore, ankle-covered shoes may be a better choice for subacute or chronic hemiplegic stroke survivors with ankle instability without foot drop than non-ankle-covered shoes and can be used as an alternative to an ankle orthosis.

This study has a few limitations. First, since there were a few number of subjects, it would be difficult to generalize the results of this study. Second, no direct comparison was made with an ankle orthosis. Therefore, it is not possible to assert only with the results of this study that putting on ankle-covered shoes would be more effective for gait improvement than not putting on shoes or putting on non-ankle-covered shoes and that it would bring about a similar effects as an ankle orthosis. Therefore, it is necessary to conduct further follow-up studies to investigate this matter.

\section{Conflict of Interest}

The authors declared no potential conflicts of interest with respect to the authorship and/or publication of this article.

\section{References}

1. Hsieh MJ, Tang SC, Chiang WC, Tsai LK, Jeng JS, Ma MH; Taipei EMS Stroke Collaborative Group. Effect of prehospital notification on acute stroke care: a multicenter study. Scand J Trauma Resusc Emerg Med 2016;24:57.

2. KOSIS. The number of stroke patients (by region, by month) [Internet]. Daejeon: Statistics Korea, 2017 [cited 2017 Dec 15]. Available from: http://kosis.kr/statHtml/statHtml.do?orgId=411 \&tblId=DT_41104_221\&conn_path=I2.

3. Karthikbabu S, Chakrapani M, Ganesan S, Ellajosyula R. Relationship between pelvic alignment and weight-bearing asymmetry in community-dwelling chronic stroke survivors. J Neurosci Rural Pract 2016;7(Suppl 1):S37-40.

4. Kim SS, Lee HJ, You YY. Effects of ankle strengthening exercises combined with motor imagery training on the timed up and go test score and weight bearing ratio in stroke patients. J Phys Ther Sci 2015;27:2303-5.

5. Balaban B, Tok F. Gait disturbances in patients with stroke. PM R 2014;6:635-42.

6. Böhm H, Matthias H, Braatz F, Döderlein L. Effect of floor reaction ankle-foot orthosis on crouch gait in survivors with cerebral palsy: what can be expected? Prosthet Orthot Int 2017. doi: 10.1177/0309364617716240. [Epub ahead of print]

7. Zissimopoulos A, Fatone S, Gard S. The effect of ankle-foot orthoses on self-reported balance confidence in persons with chronic poststroke hemiplegia. Prosthet Orthot Int 2014;38:14854. 
8. Lee KM, Byun JH. The development of ankle control elastic band for improvement in walking of hemiplegic patients. $\mathrm{J}$ Korean Acad Rehabil Med 2005;29:303-8.

9. Chaudhuri S, Aruin AS. The effect of shoe lifts on static and dynamic postural control in individuals with hemiparesis. Arch Phys Med Rehabil 2000;81:1498-503.

10. Rodriguez GM, Aruin AS. The effect of shoe wedges and lifts on symmetry of stance and weight bearing in hemiparetic individuals. Arch Phys Med Rehabil 2002;83:478-82.

11. Choi KJ. The analyses of biomechanical differences between general walking shoe and mBT functional walking shoe $[\mathrm{PhD}$ dissertation]. Seoul: Sungkyunkwan University; 2003.

12. Elizabeth EM, Katherine KW, Daniel EL, Heather LN, Rachael ED. The effect of minimal shoes on arch structure and intrinsic foot muscle strength. J Sport Health Sci 2014;3:74-85.

13. Fuller JT, Thewlis D, Tsiros MD, Brown NAT, Buckley JD. Six-week transition to minimalist shoes improves running economy and time-trial performance. J Sci Med Sport 2017;20:111722.

14. Jung HK, Chung E, Lee BH. A comparison of the balance and gait function between children with Down syndrome and typically developing children. J Phys Ther Sci 2017;29:123-7.

15. Kim JM, Kim JJ, Park SY, Cha JH, Kim MJ, Kim JA. Walking analysis in dementia using GAITRite ambulation system. J Korean Clin Health Sci 2017;5:816-24.

16. Mauritz KH. Gait training in hemiplegia. Eur J Neurol 2002;9 Suppl 1:23-9; dicussion 53-61.

17. Rao N, Wening J, Hasso D, Gnanapragasam G, Perera P, Srigiriraju $\mathrm{P}$, et al. The effects of two different ankle-foot orthoses on gait of patients with acute hemiparetic cerebrovascular accident. Rehabil Res Pract 2014;2014:301469.

18. Leung J, Moseley A. Impact of ankle-foot orthoses on gait and leg muscle activity in adults with hemiplegia: systematic literature review. Physiotherapy 2003;89:39-55.

19. Brandstater ME, de Bruin H, Gowland C, Clark BM. Hemiplegic gait: analysis of temporal variables. Arch Phys Med Rehabil 1983;64:583-7.

20. Farmani F, Mohseni-Bandpei MA, Bahramizadeh M, Aminian G, Abdoli A, Sadeghi-Goghari M. The influence of rocker bar ankle foot orthosis on gait in patients with chronic hemiplegia. $\mathrm{J}$ Stroke Cerebrovasc Dis 2016;25:2078-82.

21. Eckhardt MM, Mulder MC, Horemans HL, van der Woude LH, Ribbers GM. The effects of high custom made shoes on gait characteristics and patient satisfaction in hemiplegic gait. Gait Posture 2011;34:543-7. 